\title{
Characterization of a new high copy Stowaway family MITE, BRAMI-1 in Brassica genome
}

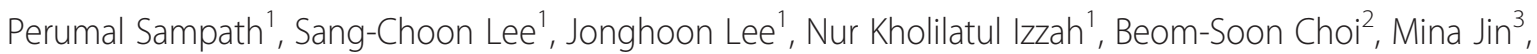
Beom-Seok Park ${ }^{3}$ and Tae-Jin Yang ${ }^{1 *}$

\begin{abstract}
Background: Miniature inverted-repeat transposable elements (MITEs) are expected to play important roles in evolution of genes and genome in plants, especially in the highly duplicated plant genomes. Various MITE families and their roles in plants have been characterized. However, there have been fewer studies of MITE families and their potential roles in evolution of the recently triplicated Brassica genome.

Results: We identified a new MITE family, BRAMI-1, belonging to the Stowaway super-family in the Brassica genome. In silico mapping revealed that 697 members are dispersed throughout the euchromatic regions of the B. rapa pseudo-chromosomes. Among them, 548 members (78.6\%) are located in gene-rich regions, less than $3 \mathrm{~kb}$ from genes. In addition, we identified 516 and 15 members in the $470 \mathrm{Mb}$ and $15 \mathrm{Mb}$ genomic shotgun sequences currently available for $B$. oleracea and $B$. napus, respectively. The resulting estimated copy numbers for the entire genomes were 1440, 1464 and 2490 in B. rapa, B. oleracea and B. napus, respectively. Concurrently, only 70 members of the related Arabidopsis ATTIRTA-1 MITE family were identified in the Arabidopsis genome. Phylogenetic analysis revealed that BRAMI-1 elements proliferated in the Brassica genus after divergence from the Arabidopsis lineage. MITE insertion polymorphism (MIP) was inspected for 50 BRAMI-1 members, revealing high levels of insertion polymorphism between and within species of Brassica that clarify BRAMI-1 activation periods up to the present. Comparative analysis of the 71 genes harbouring the BRAMI-1 elements with their non-insertion paralogs (NIPS) showed that the BRAMI-1 insertions mainly reside in non-coding sequences and that the expression levels of genes with the elements differ from those of their NIPs.

Conclusion: A Stowaway family MITE, named as BRAMI-1, was gradually amplified and remained present in over than 1400 copies in each of three Brassica species. Overall, $78 \%$ of the members were identified in gene-rich regions, and it is assumed that they may contribute to the evolution of duplicated genes in the highly duplicated Brassica genome. The resulting MIPs can serve as a good source of DNA markers for Brassica crops because the insertions are highly dispersed in the gene-rich euchromatin region and are polymorphic between or within species.
\end{abstract}

Keywords: Miniature Inverted-repeat Transposable Element (MITE), MITE insertion polymorphism (MIP), Brassica species, Evolution, BRAMI-1

\footnotetext{
* Correspondence: tjyang@snu.ac.kr

${ }^{1}$ Dept. of Plant Science, Plant Genomics and Breeding Institute, and Research Institute for Agriculture and Life Sciences, College of Agriculture and Life Sciences, Seoul National University, Seoul 151-921, Republic of Korea Full list of author information is available at the end of the article
} 


\section{Background}

The large-scale sequencing of eukaryotic genomes has revealed that transposable elements (TEs) are present ubiquitously and occupy large fractions of genomes: 5\% in yeast, $35 \%$ in rice, $45 \%$ in human, and up to $85 \%$ in maize [1-9]. TEs are classified into two classes based on their transposition mechanism. Class I mobile genetic elements, or retrotransposons, are replicated through RNA intermediates by a copy-and-paste mechanism, whereas Class II mobile genetic elements, or DNA transposons, move directly from DNA via a cut-and-paste mechanism $[1,2,10]$.

Miniature inverted-repeat transposable elements (MITEs) are Class II DNA transposons that are nonautonomous, with defective or absent of coding genes. MITEs were identified in the maize genome [11] and later found in Arabidopsis, rice, grape, mosquito, fish, bacteria and human as well as in several other genomes [1,12-15]. Due to their extremely high copy numbers, MITEs can account for a significant fraction of a eukaryotic genome (i.e. $>8 \%$ of the rice genome) even though the size of element itself is small [16]. Individual MITEs are usually less than $600 \mathrm{bp}$ and $\mathrm{A} / \mathrm{T}$ rich, with terminal inverted repeats (TIRs) and 2-11 bp target site duplication (TSD) sequences [1,10]. MITEs, which are relatively stable in the genome, are often closely associated with genic regions and thus can affect gene expression patterns $[16,17]$. Some MITEs are involved in upregulation of host genes by providing additional recognition sequences or polyA signals to host genes $[14,18,19]$. MITE insertion into regulatory regions may cause disruption or promotion of gene expression [18]. Recent studies have found that MITEs are also a source of small interfering RNA (siRNA) evolution and may play an important role in gene regulation and epigenetic mechanisms [16,20-22]. MITE transposition into a new region of the genome causes insertion polymorphisms among accessions of same species that can be useful tools for development of various markers [23,24].

The Brassicaceae family includes 338 genera and 3700 species, which serve as sources of vegetable, fodder, condiments and oil, with wide range of morphologies, such as Chinese cabbage, mustard, cabbage, broccoli, oilseed rape, and other leafy vegetables. The model plant, Arabidopsis thaliana is a close relative of the Brassica species and belongs to the same family. As a model Brassica crop, the B. rapa genome sequence spanning $256 \mathrm{Mb}$ euchromatin chromosome spaces was completed recently and released to the public [25].

Comparative analysis of Brassica species with A. thaliana has revealed up to two additional rounds of recent genome duplication: one triplication and one allopolyploidization that is the major factor responsible for the increased genome size of Brassica [25-27]. In addition, TEs also contribute to increase the genome size of the Brassica species and to genome evolution [28]. The completed genome sequence of $B$. rapa revealed that at least $39.5 \%$ of the genome contains TEs [25].

In this study, we identified a new MITE named Brassica rapa MITE (BRAMI)-1, which is present in more than 1400 copies in the genome of each of three Brassica species. We inspected its characteristics and distribution and inferred its potential involvement in the evolution of duplicated genes in the highly replicated Brassica genome. We also discovered high amounts of insertion polymorphism inter- and intra-species, which can serve a good source of genetic markers in the Brassica species.

\section{Results}

\section{Characterization of BRAMI-1 in Brassica}

We identified a 260 bp MITE in the Brassica rapa BAC clone, KBrB059A03 using MUST, a de novo program for MITE identification, and additional manual inspection. MITE characterization on B. rapa contig (KBrB059A03) using MUST yielded 291 candidate MITEs and further careful manual inspection of each candidate MITE for TIR and TSD using self-BLAST (http://blast.ncbi. nlm.nih.gov/) led to the identification of BRAMI-1. Comparison of $B R A M I-1$ against the repeat database (www.girinst.org/) showed $77 \%$ similarity to a reported Stowaway MITE, ATTIRTA-1 in A. thaliana [29]. Perfect MITE insertion was confirmed by comparing one of the representative $B$. rapa genes (Bra013859) harboring a BRAMI-1 insertion with the related empty sites in its non-insertion paralogs (NIPs) (Bra010475 and Bra019193) from B. rapa syntenic blocks and its ortholog (At4g25050) in A. thaliana (Figure 1a, b). The MITE included $33 \mathrm{bp}$ of highly conserved $\mathrm{A} / \mathrm{T}$ rich $(>69 \%)$ TIRs and was flanked with a unique dinucleotide TA target site duplication (TSD), which are distinct characteristics of the Stowaway super-family MITEs (Figure 1b, c). The secondary structure of the MITE was predicted using mfold (Figure 1d), which showed a potential DNA hairpin-like secondary structure.

BLASTn searches revealed a total of 697 BRAMI-1 elements in the $256 \mathrm{Mb} \mathrm{B}$. rapa genome sequence. In silico mapping of these elements on the $B$. rapa pseudochromosomes showed that they were evenly distributed in the euchromatin regions of the $B$. rapa genome (Figure 2). The physical positions of the 697 BRAMI-1 elements in the $B$. rapa genome are listed in Additional file 1. On average, $70 B R A M I-1$ elements were found on each pseudo-chromosome. MITE density analysis (chromosome size/no. of MITEs per chromosome) shows chromosome $3(31.72 \mathrm{Mb})$, which is the second largest in size, has the high MITE density (MITE/ $0.28 \mathrm{Mb}$ ), while the largest chromosome 9 (37.12 Mb) had the less MITE density (MITE/0.44 Mb). 
(a)

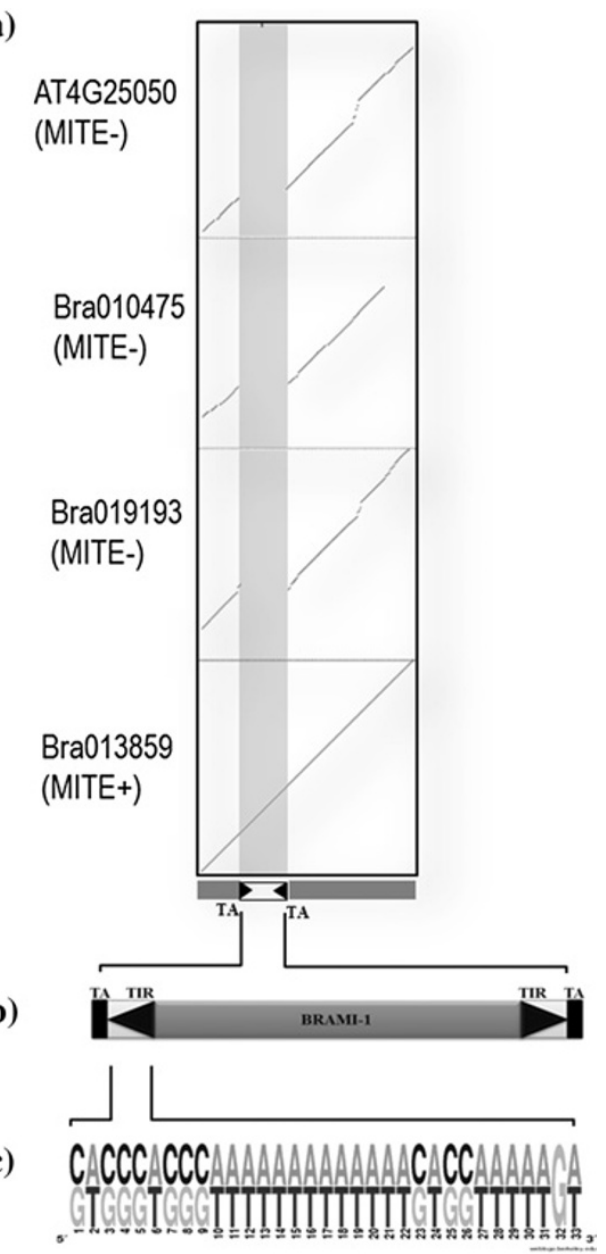

(d)

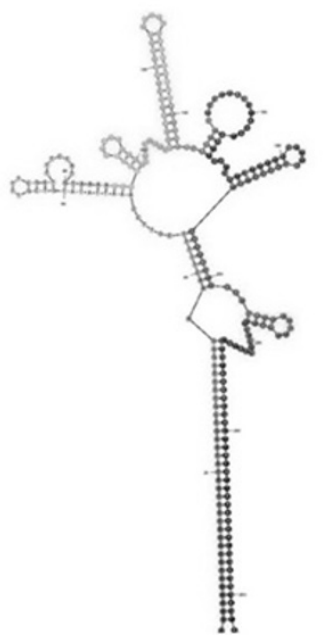

Figure 1 Identification and characterization of the BRAMI-1 elements. (a) Dotplot analysis of Bra013859 and the related empty sites in its two non-insertion paralog (NIP) genes, Bra019193 and Bra010475 from B. rapa and its orthologue At4g25050 from A. thaliana (b) The structure of BRAMI-1 showing its characteristic properties, TA Target site duplication (c) Conserved 33 bp TIR sequences shown by Weblogo analysis (d) Hypothetical secondary structure and expected loop formation predicted by mfold.
We found 516 and 15 copies in $470 \mathrm{Mb}$ of B. oleracea and $15 \mathrm{Mb}$ of $B$. napus shotgun sequences, respectively. Based on this, the total numbers of the BRAMI-1 MITE members were estimated as 1440,1464 and 2490 in the whole genomes of $B$. rapa, B. oleracea and B. napus, respectively (Table 1). By contrast, in A. thaliana we found only 70 copies of ATTIRTA-1, the closest Arabidopsis relative of BRAMI-1. Simple comparison revealed that the copy numbers of these MITEs in Brassica genomes are 20-35 times more than that of Arabidopsis.

\section{Phylogenetic analysis of the BRAMI-1 elements}

Phylogenetic analysis was conducted for 528 nearly intact MITE members that have $>80 \%$ similarity to BRAMI-1: 401 members from $B$. rapa, 123 from B. oleracea, and four from $B$. napus. In addition, 34 ATTIRTA-1 members from $A$. thaliana were included. The ATTIRTA-1 members formed a separate clade from the Brassica members, and they were very diverse among themselves. By contrast, BRAMI-1 members from the three Brassica species were highly conserved and were interspersed with each other (Figure 3) indicating they were rapidly amplified in the Brassica genome after divergence from Arabidopsis. Due to their high sequence similarity, we could not distinguish any separate clades for the BRAMI-1 family members in the Brassica species.

$B R A M I-1$ insertion in genic regions of the $B$. rapa genome We inspected the insertion sites of the 697 BRAMI-1 elements in the $B$. rapa genome using the annotated $B$. rapa genome database [31]. The analysis showed that 548 members $(78.6 \%)$ were located in gene-rich regions, less than $3 \mathrm{~kb}$ from genes. Among them, 71 (10.2\%) were inside the gene structure, specifically in introns, and 281 $(40.3 \%)$ were within less than $1 \mathrm{~kb}$ of a gene (Table 2 ).

We closely inspected the 71 genic insertions by comparing with their NIPs from triplicated chromosomal blocks. Similar numbers of insertions were identified in tri-, di-, and mono-copy genes (20, 26, and 24 insertions, respectively; Table 3 ) indicating that multi-copy genes did not preferentially contain $B R A M I-1$ insertions. Comparison of genes containing the BRAMI-1 insertion and their NIPs genes in the triplicated blocks revealed that all of the elements resided in intronic regions.

For example, Bra024324 gene was annotated as having 13 exons and included the BRAMI-1 insertion in the $7^{\text {th }}$ intron. Its two NIPs (Bra031904, Bra037793) and its Arabidopsis ortholog (At5g64740, CELLULOSE SYNTHASE 6) have similar structures in which the exonic regions share conserved sequences with Bra024324 (Figure 4a). Another gene, Bra010574, which has the $B R A M I-1$ insertion in $5^{\text {th }}$ intron, showed conserved CDS sequences without any change of gene structure compared to its NIPs (Bra011704) and its Arabidopsis ortholog 


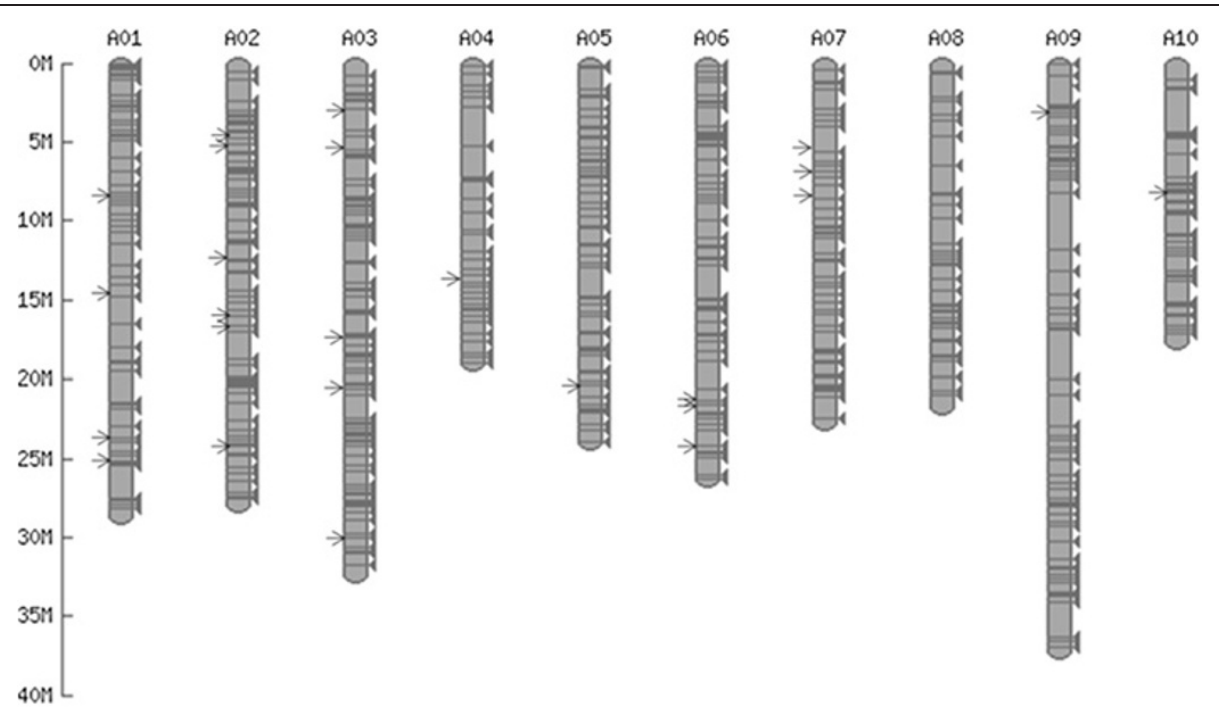

Figure $\mathbf{2}$ In silico mapping of BRAMI-1 elements in $256 \mathrm{Mb}$ of B. rapa pseudo-chromosomes. Arrows indicate the positions of the 25 members used for MIP analysis. The exact physical positions of the 697 BRAMI-1 members are listed in Additional file 1.

(At4g36760, 15 ORF, N-1-NAPHTHYLPHTHALAMIC ACID BINDING PROTEIN) (Figure 4b).

\section{Transcriptional changes of $B$. rapa genes containing BRAMI-1 insertions}

Even though most of the BRAMI-1 insertions were found in introns or UTRs, some modification of gene expression might still be mediated by BRAMI-1. Therefore, we analyzed expression level changes by comparison to NIPs using a $B$. rapa microarray database. Among the 46 multicopy genes with BRAMI-1 insertions (20 tri-copy genes and 26 di-copy genes), only six were present along with their NIPs in the microarray database. Of the six genes with BRAMI-1 insertions, only Bra039627 showed similar expression to that of its NIPs, regardless of stress treatments. One gene, Bra024324, showed decreased expression and four genes, Bra027185, Bra039330, Bra034678, and Bra010574, showed increased expression compared to that of their NIPs (Figure 5).
The expression of Bra024324, which contains a BRAMI-1 insertion, was severely decreased compared to that of its NIPs, Bra031904 and Bra037793, under normal conditions and also under the four stress treatment conditions, indicating that Bra024324 gene expression was maintained at a very low level even though the $B R A M I-1$ insertion did not affect exons (Figure 5a). By contrast, expression of Bra010574, with a BRAMI-1 insertion, was more than 3-fold higher than expression of its NIP Bra011704 under control and all four treatment conditions (Figure 5b).

\section{Survey of MITE insertion polymorphisms (MIPs) and estimation of activation dates}

To analyze $B R A M I-1$ 's transposition activity and insertion time, we designed 50 MIP primers, 25 for B. rapa and 25 for $B$. oleracea, from the flanking regions of the $B R A M I-1$ insertions, especially insertions in genic regions (Additional file 2). The positions of the $25 \mathrm{~B}$. rapa MIPs are denoted as arrows on the in silico map (Figure 2).

Table 1 Summary of observed and predicted copy numbers of the BRAMI-1 elements in Brassica relatives

\begin{tabular}{ccccc}
\hline MITE & & BRAMI-1 & ATTIRTA-1 \\
\cline { 2 - 5 } & B. oleracea & B. rapa & B. napus & A. thaliana \\
\hline Database type & GSS & Pseudo-chromosomes & GSS & $15 \mathrm{Mb}$ \\
Database size & $470 \mathrm{Mb}$ & $256 \mathrm{Mb}$ & 11 & $119 \mathrm{Mb}$ \\
Total copies & 399 & 697 & 401 & $700 \mathrm{bp}$ \\
(>80\% similarity) & 123 & $\mathrm{~N} / \mathrm{A}$ & $1132 \mathrm{Mb}$ & 34 \\
Average length of the GSS sequence & $700 \mathrm{bp}$ & $529 \mathrm{Mb}$ & 2490 & $157 \mathrm{Mb}$ \\
Estimated Genome Size [30] & $696 \mathrm{Mb}$ & 1440 & 44 \\
\hline
\end{tabular}

BLASTn was performed at the local database (http://imcrop.snu.ac.kr). N/A: not applicable. 
Figure 3 Phylogenetic tree of BRAMI-1 elements from Brassica species and ATTIRTA-1 from A. thaliana. Relatively intact MITE members showing $80 \%$ similarity to the characteristic MITE structure were used for the analysis. A total of 528 BRAMI-1 members including 401, 123, and 4 from B. rapa (red), B. oleracea (blue), and B. napus (black), respectively, and 34 ATTIRTA-1 members (green) were compared. Sequence alignment was conducted using ClustalW and then the phylogenetic tree was generated using the neighbor joining method with 500 bootstrap replicates.

Almost all of the primer pairs revealed polymorphisms (48 in 50 pairs; 96\%) among seven accessions belonging to three Brassica species, indicating that the BRAMI-1 members have been continuously activated during diversification of the Brassica genome. Moreover, there was high polymorphism within species, with seven (14\%), six (12\%), and ten (20\%) polymorphisms among two accessions of $B$. napus, two accessions of B. rapa, and three accessions of B. oleracea, respectively.

We grouped the 50 MIPs into three different groups: BRAMI-1 Bs (common to both species), Br (B. rapa-unique), and Bo (B. oleracea-unique), to deduce the tentative insertion times (Figure 6a). The Br and Bo MIPs were further classified into two subgroups, -I and -II, based on the presence or absence of the insertion in their allopolyploid species B. napus. Among the 25 B. rapa MIPs, 3, 17 , and 5 were $\mathrm{Bs}, \mathrm{Br}-\mathrm{I}$, and $\mathrm{Br}-\mathrm{II}$ type insertions, respectively, and among the $25 \mathrm{~B}$. oleracea MIPs, 6, 18, and 1 were Bs, Bo-I, and Bo-II types, respectively. Overall, $18 \%$ were shared in the Brassica genus, and $82 \%$ were species-unique insertions (Figure 6b).

Phylogenetic analysis based on the 50 MIP profiles revealed four distinct clusters at the 0.30 genetic similarity coefficient level (Additional file 3). Arabidopsis was separated from Brassica accessions with a genetic similarity coefficient of 0.16. Three Brassica species each formed a distinct cluster with two or three accessions belonging to each species, corresponding well with the phylogeny of Brassica species. Each MIP reflects the insertion time at that genomic position and thus MIP-based genotyping and phylogenetic analysis will be a good tool for study of genetic diversity in the Brassica genus. We also confirmed that the MIPs are clearly distinguishable on agarose gels, heritable and reproducible, characteristics beneficial as DNA markers. A MIP between two B. oleracea accessions, Bo-19, segregated according to a normal Mendelian 1: 2: 1 ratio in a survey of $94 \mathrm{~F}_{2}$ progeny of a cross between the two accessions (Additional file 4).

\section{Discussion}

Structure, distribution and evolution of $B R A M I-1$ in the $B$. rapa genome

$B R A M I-1$ exhibits the basic characteristics of conventional Stowaway-like MITEs, which include small size, 
Table 2 Summary of the insertion positions of 697 BRAMI-1 elements in the B. rapa genome

\begin{tabular}{lcc}
\hline Insertion position & Number of elements & Percentage of elements \\
\hline Gene & 71 & 10.2 \\
Near Genic Regions $(<1 \mathrm{~kb})^{\mathrm{a}}$ & 281 & 40.3 \\
Near Genic Regions $(1 \mathrm{~kb} \text { to }<2 \mathrm{~kb})^{\mathrm{a}}$ & 134 & 19.2 \\
Near Genic Regions $(2 \mathrm{~kb} \text { to }<3 \mathrm{~kb})^{\mathrm{a}}$ & 62 & 8.9 \\
Intergenic Region $(>3 \mathrm{~kb})^{\mathrm{a}}$ & 149 & 21.4 \\
Total & 697 & 100.0 \\
\hline
\end{tabular}

${ }^{\mathrm{a}}$ Distance from nearest gene.

TIRs, and TSDs, and also possesses a potential DNA hairpin-like secondary structure. BRAMI-1 elements have a highly conserved 33 bp TIR region that is rich in A $+\mathrm{T}$ nucleotides (>69\%) and a 194 bp internal region. In plants, most MITEs are classified as either Touristlike or Stowaway-like. Tourist-like MITEs are regarded as deletion derivatives of full-length autonomous TEs, such as mPing derived from Pong and PIF [13,32,33]. The origin of Stowaway-like MITEs is unclear due to the lack of sufficient sequence similarity to known autonomous TEs [1,34]. However, numerous Stowawaylike MITEs can be cross-mobilized by distantly related Mariner-like elements (MLEs) to generate high copy numbers [13,35]. However, we could not identify the trans-acting autonomous element for the $B R A M I-1$ elements in this study.

\section{Rapid amplification of BRAMI-1 elements in the Brassica genus}

The genus Brassica is an excellent model plant to study polyploidization-mediated genome evolution because allotetraploid species like $B$. juncea, $B$. napus, and $B$. carinata evolved very recently from the three diploid species $B$. rapa, B. oleracea, and B. nigra, and even the diploid Brassica species have triplicated genome features that arose approximately 13 million years ago (MYA) $[26,27,36]$. The estimated copy numbers of the BRAMI-1 elements were similar in two closely related Brassica species: 1440 and 1464 in B. rapa and B. oleracea, respectively supporting that $B R A M I-1$ elements were actively amplified in both Brassica species [27,36,37]. This is the first MITE found to exhibit very high copy numbers in Brassica, although one medium copy number Brassica Stowaway MITE, named Brasto, was recently characterized [38].

BRAMI-1 shares $77 \%$ similarity with the $A$. thaliana MITE ATTIRTA-1, suggesting that they evolved from a common ancestor of the Brassica and Arabidopsis lineage. Phylogenetic analysis revealed that ATTIRTA-1 and $B R A M I-1$ elements have clearly different evolutionary histories. The ATTIRTA-1 elements showed a high amount of variation even though their copy numbers were small compared to those of the BRAMI-1 members, indicating that the ATTIRTA-1 members were maintained in the Arabidopsis genome without further amplification after the split from the Brassica lineage 13-17 MYA [27,36]. By contrast, members derived from $B$. rapa (red), B. oleracea (blue), and B. napus (black) are highly conserved and interrelated with each other, demonstrating that the members were actively amplified in the Brassica lineage after divergence from Arabidopsis (Figure 3). This is consistent with a report showing highly active TE amplification in B. oleracea [28]. We assume that several transpositional bursts may have been responsible for the amplification of the BRAMI-1 members in the Brassica lineage [16,39,40].

\section{The putative role of $B R A M I-1$ in $B$. rapa genome evolution} There have been many reports of MITEs involved in the evolution of genes and genomes. MITEs are often inserted in genic regions such as promoter regions, UTRs, introns, or exons and can influence the expression of genes $[1,2,16,19,34]$. MITE insertion into the various functional regions of a gene can modify its transcriptional activity, cause silencing, and up- or down-regulation of gene expression [34,41]. We found 697 BRAMI-1 elements were dispersed across the whole genome (Figure 2). A total of 626 members (90\%) were identified in $177 \mathrm{Mb}$ of intergenic spaces and 71 members (10\%) were identified in $79 \mathrm{Mb}$ of gene spaces in the $256 \mathrm{Mb} \mathrm{B}$. rapa pseudo chromosome sequences. Among the 697 elements, 548 members $(78.6 \%)$ were located within $3 \mathrm{~kb}$ of genic regions and all the 71 copies found in genic regions were resided in introns. The $33 \mathrm{Mb}$ intronic regions exhibited $65 \% \mathrm{~A}+\mathrm{T}$ composition, which was much higher than that of $46 \mathrm{Mb}$ exonic regions (54\% $\mathrm{A}+\mathrm{T}$ composition). This insertion target site preference for non-coding sequences of genic regions is similar to the insertion preference of $m$ Ping in rice, which is more often found in $\mathrm{A}+\mathrm{T}$ rich noncoding sequence than in $G+C$ rich exonic regions [19].

We showed that BRAMI-1 insertion might be one of the causal forces for modification of gene expression. When we compared the expressions of several genes harboring $B R A M I-1$ within their genic regions with those of NIPs, most of the genes with BRAMI-1 insertions showed different expression patterns than their NIP counterparts (Figure 5). Comparison of microsynteny between regions 
Table 3 Insertion positions and names of the 71 genes harboring BRAMI-1 elements in intronic regions and list of their orthologous genes in Arabidopsis and NIPs in the triplicated blocks of the B. rapa genome

\begin{tabular}{|c|c|c|c|c|c|c|c|c|}
\hline & \multirow{2}{*}{$\begin{array}{l}\text { MITE } \\
\text { No }\end{array}$} & \multirow{2}{*}{$\begin{array}{l}\text { Chr } \\
\text { No }\end{array}$} & \multirow{2}{*}{$\begin{array}{l}\text { MITE } \\
\text { start }\end{array}$} & \multirow{2}{*}{$\begin{array}{l}\text { MITE } \\
\text { end }\end{array}$} & \multirow{2}{*}{$\begin{array}{l}\text { Ortholog from } \\
\text { A. thaliana }\end{array}$} & \multicolumn{3}{|c|}{ Triplicated blocks in B. rapa ${ }^{2}$} \\
\hline & & & & & & LF & MF1 & MF2 \\
\hline \multirow[t]{20}{*}{ THREE COPY GENES } & 23 & $\mathrm{~A} 01$ & 8110084 & 8109821 & At4g25050 & Bra013859 & Bra019193 & Bra010475 \\
\hline & 83 & $\mathrm{~A} 02$ & 4177719 & 4177456 & At5g17300 & Bra008563 & Bra006394 & Bra023610 \\
\hline & 176 & $\mathrm{~A} 03$ & 5778627 & 5778365 & At5g55050 & Bra002937 & Bra035549 & Bra028994 \\
\hline & 188 & A03 & 8910390 & 8910639 & At2g37940 & Bra005148 & Bra017148 & Bra000029 \\
\hline & 219 & A03 & 17370042 & 17370087 & At3g15820 & Bra027205 & Bra021138 & Bra001607 \\
\hline & 220 & $\mathrm{~A} 03$ & 17370087 & 17370331 & At3g15820 & Bra027205 & Bra021138 & Bra001607 \\
\hline & 299 & $\mathrm{~A} 04$ & 13405505 & 13405756 & At2g30110 & Bra018338 & Bra021611 & Bra022779 \\
\hline & 303 & $\mathrm{~A} 04$ & 14136797 & 14136887 & At2g31500 & Bra018236 & Bra021727 & Bra022844 \\
\hline & 346 & A05 & 7674854 & 7674713 & At2g29980 & Bra018348 & Bra021599 & Bra022767 \\
\hline & 347 & A05 & 7674974 & 7675006 & At2g29980 & Bra018348 & Bra021599 & Bra022767 \\
\hline & 349 & A05 & 8733844 & 8733992 & At4g04640 & Bra029511 & Bra000802 & Bra018503 \\
\hline & 368 & A05 & 16917820 & 16918070 & At3g20770 & Bra035746 & Bra023927 & Bra001802 \\
\hline & 425 & A06 & 14973843 & 14973752 & At5g64740 & Bra024324 & Bra037793 & Bra031904 \\
\hline & 443 & A06 & 22195786 & 22196049 & At3g28050 & Bra025321 & Bra033037 & Bra039062 \\
\hline & 450 & A06 & 24123977 & 24123793 & At2g01430 & Bra024888 & Bra026666 & Bra017451 \\
\hline & 452 & A06 & 24666391 & 24666142 & At5g46630 & Bra025009 & Bra022052 & Bra017537 \\
\hline & 473 & A07 & 8537339 & 8537527 & At1g22340 & Bra031388 & Bra012324 & Bra016424 \\
\hline & 474 & A07 & 9114152 & 9114414 & At1g20670 & Bra025837 & Bra012243 & Bra016456 \\
\hline & 566 & A08 & 20712404 & 20712596 & At1g07920 & Bra018669 & Bra031602 & Bra030707 \\
\hline & 654 & $\mathrm{~A} 10$ & 1570075 & 1569913 & At1g06080 & Bra015473 & Bra032437 & Bra030638 \\
\hline \multirow[t]{22}{*}{ TWO COPY GENES } & 46 & $\mathrm{~A} 01$ & 18882651 & 18882770 & At5g52140 & Bra028293 & Bra022579 & - \\
\hline & 55 & $\mathrm{~A} 01$ & 23626196 & 23626459 & At3g16180 & Bra027185 & Bra021168 & - \\
\hline & 61 & $\mathrm{~A} 01$ & 25069244 & 25069502 & At3g02180 & - & Bra021476 & Bra001035 \\
\hline & 87 & $\mathrm{~A} 02$ & 5079784 & 5080047 & At5g20540 & - & Bra006563 & Bra020109 \\
\hline & 113 & A02 & 9852697 & 9852644 & At1g66370 & Bra004162 & Bra039763 & - \\
\hline & 153 & $\mathrm{~A} 02$ & 25486260 & 25486523 & At5g23940 & Bra009716 & - & Bra029388 \\
\hline & 168 & $\mathrm{~A} 03$ & 2298612 & 2298349 & At5g12420 & - & Bra006160 & Bra023377 \\
\hline & 200 & $\mathrm{~A} 03$ & 11193078 & 11192830 & At2g47460 & Bra004456 & - & Bra000453 \\
\hline & 234 & A03 & 20936196 & 20936267 & At5g23260 & Bra013028 & Bra026507 & Bra029365 \\
\hline & 235 & A03 & 20936271 & 20936494 & At5g23260 & Bra013028 & Bra026507 & Bra029365 \\
\hline & 249 & $\mathrm{~A} 03$ & 24785451 & 24785715 & At4g22950 & - & Bra019343 & Bra020826 \\
\hline & 319 & $\mathrm{~A} 04$ & 18584148 & 18584406 & At2g45550 & Bra004921 & Bra039330 & - \\
\hline & 444 & A06 & 22352521 & 22352784 & At3g27640 & Bra025293 & - & Bra039073 \\
\hline & 460 & A07 & 1577014 & 1576769 & At2g18230 & Bra039627 & - & Bra037229 \\
\hline & 467 & A07 & 6402416 & 6402153 & At1g29120 & - & Bra030121 & Bra010851 \\
\hline & 490 & A07 & 12392864 & 12392917 & At3g57530 & Bra007334 & - & Bra003287 \\
\hline & 536 & A08 & 12108300 & 12108552 & At4g35150 & - & Bra017699 & Bra034678 \\
\hline & 545 & A08 & 15271728 & 15271631 & At4g36760 & Bra011704 & - & Bra010574 \\
\hline & 596 & A09 & 8214071 & 8213980 & At1g61890 & Bra027073 & Bra028379 & - \\
\hline & 597 & A09 & 8214185 & 8214078 & At1g61890 & Bra027073 & Bra028379 & - \\
\hline & 604 & A09 & 16501688 & 16501868 & At5g46350 & Bra025021 & Bra022033 & Bra017561 \\
\hline & 605 & A09 & 16667316 & 16667053 & At5g46040 & - & Bra022016 & Bra017582 \\
\hline
\end{tabular}


Table 3 Insertion positions and names of the 71 genes harboring BRAMI-1 elements in intronic regions and list of their orthologous genes in Arabidopsis and NIPs in the triplicated blocks of the B. rapa genome (Continued)

\begin{tabular}{|c|c|c|c|c|c|c|c|c|}
\hline & 606 & A09 & 16682960 & 16683223 & At5g46040 & - & Bra022016 & Bra017582 \\
\hline & 608 & A09 & 19871591 & 19871427 & At1g32780 & Bra023290 & Bra010185 & - \\
\hline & 615 & A09 & 24192808 & 24192545 & At1g23380 & Bra024593 & - & Bra016348 \\
\hline & 666 & A10 & 8067999 & 8067852 & At5g57655 & Bra002710 & Bra020426 & - \\
\hline & 670 & A10 & 8789727 & 8789464 & At5g59340 & Bra002576 & Bra020321 & - \\
\hline \multirow[t]{24}{*}{ ONE COPY GENES } & 40 & A01 & 14766344 & 14766081 & - & - & - & Bra029909 \\
\hline & 41 & A01 & 14767003 & 14766741 & - & - & - & Bra029909 \\
\hline & 129 & A02 & 16530545 & 16530808 & At4g01590 & - & - & Bra008554 \\
\hline & 178 & A03 & 5992774 & 5992961 & - & - & - & Bra029035 \\
\hline & 223 & A03 & 18448338 & 18448491 & At3g20360 & - & - & Bra001785 \\
\hline & 578 & A09 & 3996947 & 3996684 & At2g11810 & - & - & Bra037199 \\
\hline & 266 & A03 & 29733949 & 29734212 & - & - & Bra017680 & - \\
\hline & 268 & A03 & 30599723 & 30599787 & At4g36940 & - & Bra017808 & - \\
\hline & 472 & A07 & 7616363 & 7616100 & - & - & Bra012436 & - \\
\hline & 49 & A01 & 21397784 & 21397864 & At3g19870 & - & Bra038237 & - \\
\hline & 120 & A02 & 12485621 & 12485884 & At1g72110 & - & Bra008008 & - \\
\hline & 128 & A02 & 15935110 & 15935361 & At1g80200 & - & Bra008487 & - \\
\hline & 148 & A02 & 24005490 & 24005690 & - & - & Bra020642 & - \\
\hline & 285 & A04 & 7140471 & 7140542 & - & Bra028251 & - & - \\
\hline & 378 & A05 & 20048141 & 20048392 & - & Bra027271 & - & - \\
\hline & 445 & A06 & 22716443 & 22716194 & At3g26610 & Bra025216 & - & - \\
\hline & 501 & A07 & 16958538 & 16958801 & At1g65590 & Bra004121 & - & - \\
\hline & 513 & A07 & 20340243 & 20340188 & At1g74790 & Bra015893 & - & - \\
\hline & 655 & A10 & 4270200 & 4270396 & At1g02390 & Bra033323 & - & - \\
\hline & 656 & A10 & 4270415 & 4270484 & At1g02390 & Bra033323 & - & - \\
\hline & 657 & A10 & 4410198 & 4410053 & - & Bra033297 & - & - \\
\hline & 672 & A10 & 9364412 & 9364675 & - & Bra002467 & - & - \\
\hline & 673 & A10 & 9364744 & 9365007 & - & Bra002467 & - & - \\
\hline & 677 & A10 & 10858935 & 10858966 & - & Bra002214 & - & - \\
\hline
\end{tabular}

\footnotetext{
${ }^{\mathrm{z}}$ The triplicated chromosome blocks are denoted according to the classification of the B. rapa genome annotation databases (BRAD) [25,31]. The triplicated chromosome blocks are classified as one least fractionized block (LF) and two moderately fractionized blocks (MF1, MF2). Genes with BRAMI-1 insertion in introns are denoted in bold and their NIPs are denoted in plain letters.
}

with BRAMI-1 insertions and their non-insertion homologous genes in $B$. rapa and $A$. thaliana showed relatively conserved coding sequences but more sequence variation in introns and UTRs, including from the BRAMI-1 insertions (Figure 4). The observed changes in transcription levels might arise from $B R A M I-1$ insertions into intronic or UTR regions, similar to a recent report showing an enhancing effect of mPing near rice genes [19]. Further intensive study of whole transcriptome profiles will be necessary to address MITE effects on gene expression.

BRAMI-1 elements are active up to the present in Brassica genera

MIP patterns showing insertions specific to certain species or accessions elucidate the timing of insertion events. Among 50 MIPs, nine (18\%) BRAMI-1 elements were found in both $B$. rapa and $B$. oleracea, indicating that they were inserted into the regions before $B$. rapa and $B$. oleracea diverged from each other 4 MYA $[27,36]$. The other $41(82 \%)$ were unique to one species or the other, indicating they were inserted after the divergence of the two lineages. Among the 41 speciesspecific members, six (8\%) showed no insertion in $B$. napus (the allopolyploid product of $B$. rapa and $B$. oleracea) indicating that they inserted into each genome after allopolyplidization 0.01 MYA [36] (Figure 6). Some MIPs were found between accessions of same species, and the MIPs segregated normally in an $F_{2}$ population, opening a new window for MIP-based marker development for marker-assisted selection and 


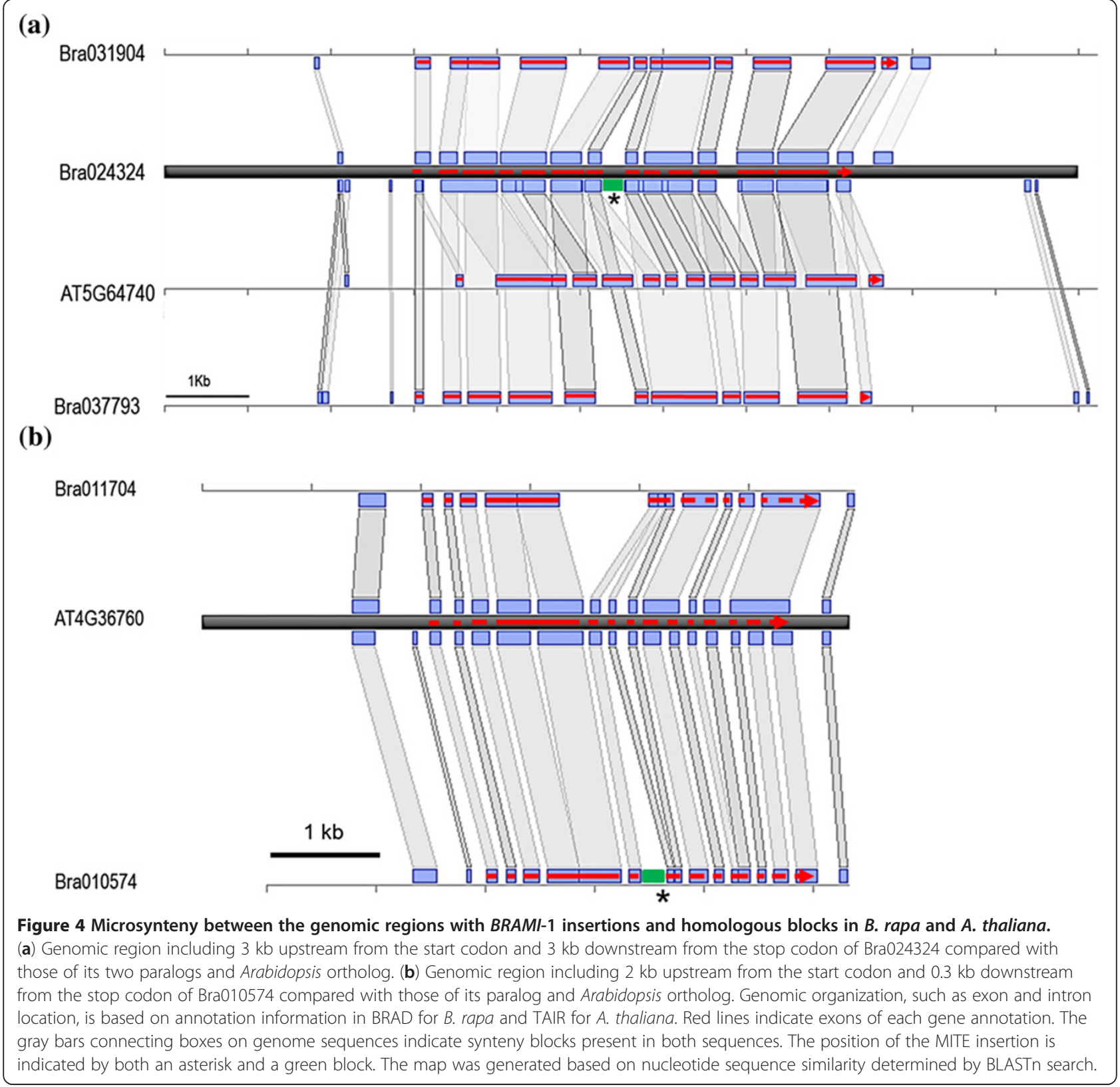

other breeding applications in Brassica crops. Overall, the MIPs revealed that BRAMI-1 elements were gradually inserted into the Brassica genome during various events and remained active up to the present.

\section{Conclusions}

We characterized a high copy Stowaway family MITE, named as BRAMI-1, in three Brassica crops and showed its putative role in the evolution of the highly duplicated Brassica genome based on comparative genomics analysis. MIP analysis revealed that the BRAMI-1 elements were dispersed into whole Brassica genome by gradual amplification. We also propose effective utilization of the elements as DNA markers for breeding and evolution of duplicated genes.

\section{Methods}

Identification and characterization of BRAMI-1

We analyzed a repeat-rich $B$. rapa BAC clone sequence, KBrB059A03 (AC189406), to find high copy repeat elements using MUST, a de novo program for MITE analysis, with the default parameters [42]. The BAC clone contained $139 \mathrm{~kb}$ of highly repetitive sequence. The structure of the TIRs was analyzed using weblogo [43]. The hypothetical DNA hairpin-like structure was predicted using the mfold application [44]. 


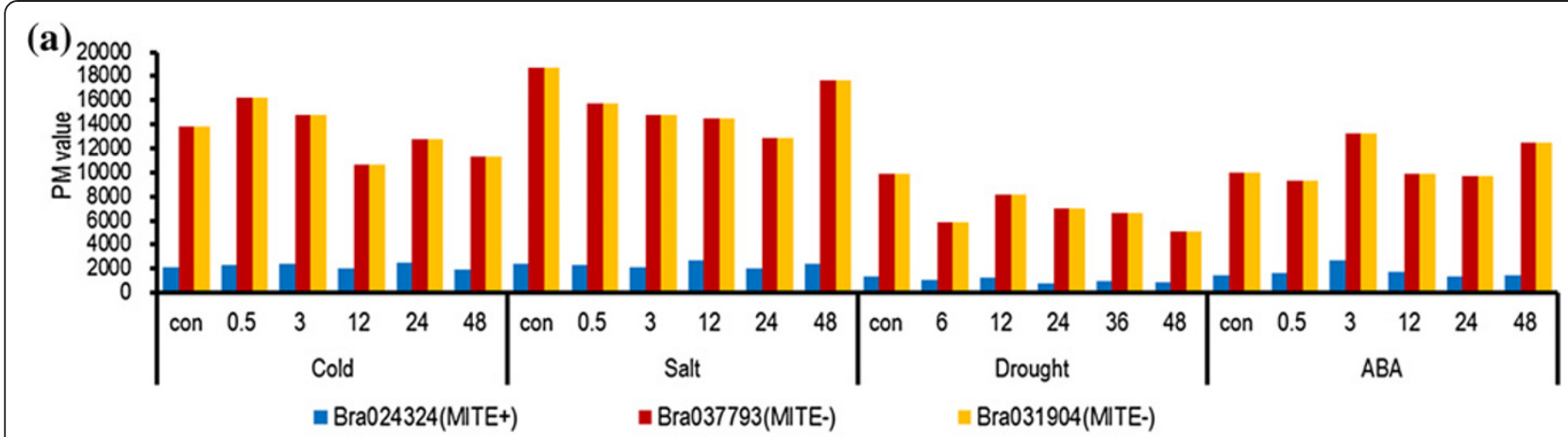

(b)

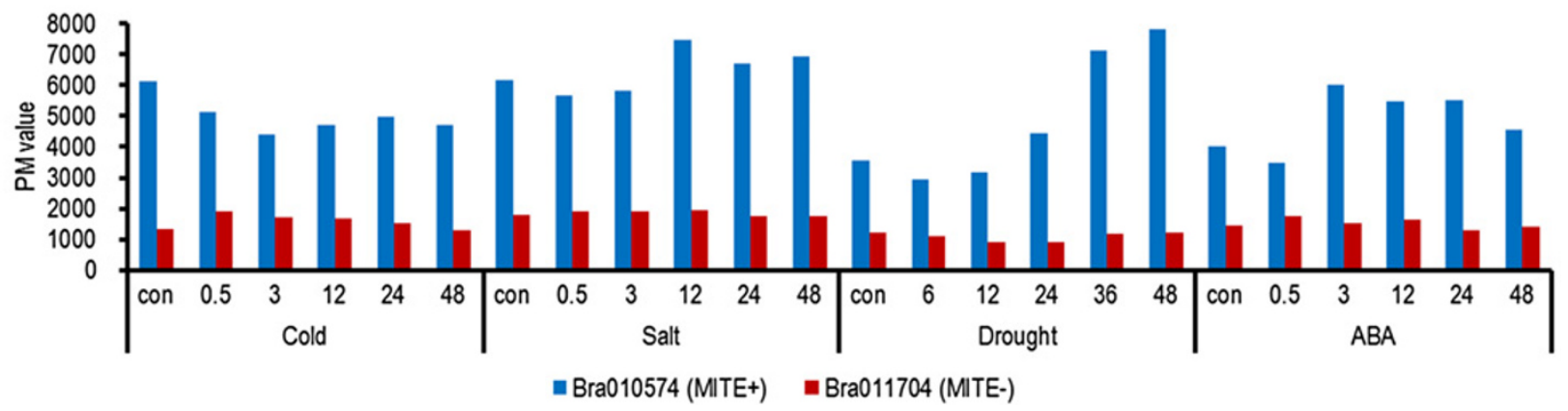

Figure 5 Comparison of expression profiles between genes with BRAMI-1 insertions and their NIPs. (a) Expressions of Bra024324 and its two NIPs, Bra031904 and Bra037793, were analyzed by searching a microarray database of B. rapa treated with cold $\left(4^{\circ} \mathrm{C}\right)$, salt $(250 \mathrm{mM} \mathrm{NaCl})$, drought (air-drying), or ABA (100 MM). (b) Expression of Bra010574 and its NIP, Bra011704, were compared. MITE+ and MITE- indicate genes with the BRAMI-1 insertion and their NIPs, respectively.

The new MITE was used as a query to retrieve its family members from a local database (http://imcrop.snu.ac.kr/) that includes $256 \mathrm{Mb}$ of 10 pseudochromosome sequences from $B$. rapa, $425 \mathrm{Mb}$ of $B$. oleracea shotgun sequences, $15 \mathrm{Mb}$ of $B$. napus shotgun sequences, and the whole genome sequence of $A$. thaliana, using the approach suggested by Wicker et al. (2007) [10]. BLASTn with default parameters [45] and a threshold E-value of $1 \mathrm{E}^{-10}$ was employed to search for MITE family members. The insertion sites of 697 elements and their flanking regions were annotated using the B. rapa genome database [31].

\section{Estimation of copy number}

The copy number of BRAMI-1 in the B. rapa genome $(529 \mathrm{Mb})$ was estimated from the number of copies identified in $256 \mathrm{Mb}$ of 10 pseudo-chromosome sequences from $B$. rapa [25]. The copy numbers in the $B$. oleracea and $B$. napus genomes were estimated by considering the hit numbers in the available genome shotgun sequences. A total of $425 \mathrm{Mb}$ of B. oleracea sequences derived from 680,894 genome shotgun sequences with an average length of $700 \mathrm{bp}$ [46] and $15 \mathrm{Mb}$ of $B$. napus shotgun sequences derived from 52,099 genome shotgun sequences (GSS) with an average length of 700 bp were downloaded from GenBank (NCBI) and used as local databases. The copy numbers of BRAMI-1 in B. oleracea and B. napus were estimated using the previously reported formula [28]: [(1/genome coverage $) / 2] \times$ number of hits $\{[1+[$ (average GSS) -TIR length $\mathrm{x} 2$ )/(average GSS length + TIR length $\mathrm{x} 2)]$ \}. Relatively intact copies with more than $80 \%$ coverage of the BRAMI-1 structure were collected from the three Brassica species for phylogenetic analysis. Multiple sequence alignment was conducted using ClustalW and phylogenetic analysis was performed based on the neighbor joining method in MEGA5 [47]. In A. thaliana, ATTIRTA-1 was the most closely related element to $B R A M I-1$, so it was included in the phylogenetic analysis. Tree topologies were evaluated using bootstrap analysis with 500 replicates for the neighborjoining method [47].

\section{Expression analysis of $B$. rapa genes with BRAM1-1 insertions}

We investigated expression modification of genes that had a MITE insertion inside of the gene structure by comparison with their syntenic paralogs using a $24 \mathrm{~K}$ microarray database (http://nabic.rda.go.kr) [48]. The microarray database represented ca. 24,000 unigenes generated from 


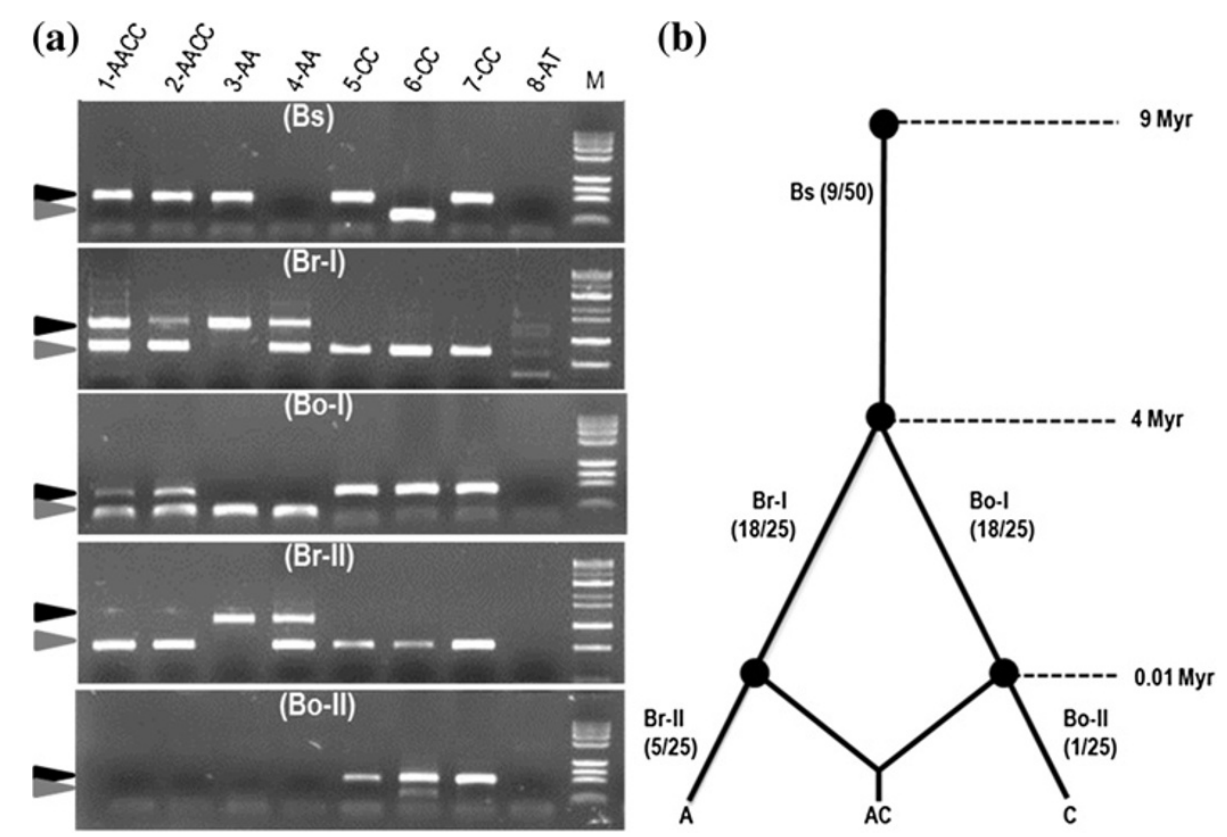

Figure 6 MITE insertion polymorphism (MIP) analysis and estimation of insertion time. MIP patterns were classified into 5 groups (Bs, Br- I, II and Bo- I, II), based on existence of MIPs between species. (a) Gel electrophoresis of five MIPs (Bo-23, Br-6, Br-3, Bo-10, Bo-21, ordered from the top, for more information on the MIP IDs refer to Additional file 2). The lane numbers (1 to 8 ) indicate plant materials used, as described in Table 1. A, C, and AC represent the genomes of B. rapa, B, oleracea, and B. napus, respectively. AT indicates A. thaliana. M, molecular size marker. The presence or absence of an insertion is denoted by a black or gray arrowhead, respectively. (b) Estimated insertion timing for the five MIP groups during the evolution of Brassica species [27,36,37].The number within the parentheses indicates the corresponding number of MITE members belonging to the particular group (based on the analysis in panel $\mathbf{a}$ ).

cDNA libraries of $B$. rapa ssp. pekinensis (inbred line 'Chiifu') and provided transcriptome profiling of changes induced by abiotic stress treatment. A given probe sequence and its ID in the microarray were searched using the coding sequence of the gene as a query. The perfect match (PM) values of probes were retrieved and processed to identify expression patterns, as described previously [48].

\section{MITE Insertion polymorphism (MIP)}

To inspect insertion polymorphisms and thus infer activation times, we used seven Brassica accessions belonging to three species and A. thaliana ecotype Columbia (Table 4). DNA was extracted from fresh leaf samples using the CTAB method [49]. In addition, a total of $94 \mathrm{~F}_{2}$ progeny from a cross between $B$. oleracea accessions $\mathrm{C} 1234$ and $\mathrm{C} 1184$ were used for segregation pattern analysis of MIPs.

We designed 50 primer pairs, 25 using shotgun sequences of $B$. oleracea (Bo 1-25) and 25 using the B. rapa pseudo-chromosome sequences (Br 1-25), from the flanking sequences of BRAM1-1 insertion sites using the Primer3 software program [50] (Additional file 2). PCR was conducted in $20 \mu \mathrm{L}$ total volume containing $10 \mathrm{ng}$ DNA, 10 pmol each primer, $250 \mu \mathrm{M}$ dNTPs, and 1 unit Taq DNA polymerase (VIVAGEN, Republic of Korea).
PCR conditions were as follows: $5 \mathrm{~min}$ at $94^{\circ} \mathrm{C}, 38$ cycles of $95^{\circ} \mathrm{C}$ for $30 \mathrm{sec}, 56^{\circ} \mathrm{C}-62^{\circ} \mathrm{C}$ for $30 \mathrm{sec}$, and $72^{\circ} \mathrm{C}$ for $60 \mathrm{sec}$, with a final extension at $72^{\circ} \mathrm{C}$ for $5 \mathrm{~min}$, using a MG96G thermo cycler (LongGene Scientific Instruments, China). PCR products were analyzed using 1\% agarose gel electrophoresis and visualized on a UV trans-illuminator after ethidium bromide staining.

For MIP marker analysis, each band was scored as ' 1 ' or ' 0 ' for presence or absence, respectively. Jaccard's similarity coefficient and a dendrogram of the genetic relationship according to Unweighted Pair Group Method with Arithmetic Average (UPGMA) analysis were determined by the

Table 4 Plant materials used for MIP analysis

\begin{tabular}{llll}
\hline & Genome & Species & Accessions (cultivars) \\
\hline 1 & AACC & B. napus & Tapidor \\
2 & AACC & B. napus & Ningyou7 \\
3 & AA & B. rapa & Chiifu \\
4 & AA & B. rapa & Kenshin \\
5 & CC & B. oleracea & C1234 \\
6 & CC & B. oleracea & C1184 \\
7 & CC & B. oleracea & C1235 \\
8 & AT & A. thaliana & Columbia \\
\hline
\end{tabular}


NTSYS-pc program (Numerical Taxonomy \& Multivariate Analysis System) [51].

\section{Additional files}

\section{Additional file 1: Physical position of the 697 members in the $B$. rapa genome.}

\section{Additional file 2: List of Primers used for MITE insertion} polymorphism analysis.

Additional file 3: Phylogenetic analysis of MIPs. Dendrogram based on Jaccard's similarity coefficient of 50 MIPs among eight genotypes constructed using the UPGMA method.

Additional file 4: MIP survey of $94 \mathrm{~B}$. oleracea $F_{2}$ plants from a cross between parental lines C1234 (P1) and C1184 (P2). The primer MIP-M1-19 was used and a ratio of 22:50:21 was observed for genotypes P1: P1/P2 : P2

\section{Competing interests}

The authors declare that they have no competing interests.

\section{Authors' contribution}

PS and TJY initiate the research. PS, SCL, JL, and NKI carried out the molecular experiments, interpreted the results. PS and BSC performed the bioinformatics analyses. MJ and BSP provided suggestion for the manuscript preparation and writing. PS and TJY wrote the manuscript. All authors critically read and approved the final version of the manuscript.

\section{Acknowledgments}

This work was supported by the Technology Development Program (No. 309008-05) for Agriculture and Forestry, Ministry of Food, Agriculture, Forestry and Fisheries, Republic of Korea and and a grant from the Next-Generation BioGreen 21 Program (No. PJ0090762012), Rural Development Administration, Republic of Korea. Perumal Sampath is supported by a Korean Government Scholarship (KGSP) 2008 from the National Institute for International Education (NIIED), Ministry of Education, Science, and Technology, Republic of Korea.

\section{Author details}

${ }^{1}$ Dept. of Plant Science, Plant Genomics and Breeding Institute, and Research Institute for Agriculture and Life Sciences, College of Agriculture and Life Sciences, Seoul National University, Seoul 151-921, Republic of Korea.

${ }^{2}$ National Instrumentation Center for Environmental Management, College of Agriculture and Life Sciences, Seoul National University, Seoul 151-921, Republic of Korea. ${ }^{3}$ National Academy of Agricultural Science, Rural Development Administration, 150 Suinro, Suwon 441-707, Republic of Korea.

\section{Received: 12 September 2012 Accepted: 18 March 2013}

Published: 2 April 2013

\section{References}

1. Feschotte $\mathrm{C}$, Jiang N, Wessler SR: Plant transposable elements: where genetics meets genomics. Nat Rev Genet 2002, 3(5):329-341.

2. Feschotte $C$ : Transposable elements and the evolution of regulatory networks. Nat Rev Genet 2008, 9(5):397-405.

3. Haberer G, Young S, Bharti AK, Gundlach H, Raymond C, Fuks G, Butler E, Wing RA, Rounsley S, Birren B, et al: Structure and architecture of the maize genome. Plant Physio/ 2005, 139(4):1612-1624

4. Lander ES, Linton LM, Birren B, Nusbaum C, Zody MC, Baldwin J, Devon K, Dewar K, Doyle M, FitzHugh W, et al: Initial sequencing and analysis of the human genome. Nature 2001, 409(6822):860-921.

5. Martiel JL, Blot M: Transposable elements and fitness of bacteria. Theor Popul Biol 2002, 61(4):509-518.

6. Morrell PL, Buckler ES, Ross-lbarra J: Crop genomics: advances and applications. Nat Rev Genet 2011, 13(2):85-96.

7. SanMiguel P, Gaut BS, Tikhonov A, Nakajima Y, Bennetzen JL: The paleontology of intergene retrotransposons of maize. Nat Genet 1998, 20(1):43-45
8. Schnable PS, Ware D, Fulton RS, Stein JC, Wei F, Pasternak S, Liang C, Zhang J, Fulton L, Graves TA, et al: The B73 maize genome: complexity, diversity, and dynamics. Science 2009, 326(5956):1112-1115.

9. Matsumoto TWJ, Kanamori $\mathrm{H}$, et al: The map-based sequence of the rice genome. Nature 2005, 436(7052):793-800.

10. Wicker T, Sabot F, Hua-Van A, Bennetzen JL, Capy P, Chalhoub B, Flavell A, Leroy $P$, Morgante $M$, Panaud $O$, et al: A unified classification system for eukaryotic transposable elements. Nat Rev Genet 2007, 8(12):973-982.

11. Bureau TE, Wessler SR: Tourist: a large family of small inverted repeat elements frequently associated with maize genes. Plant Cell 1992, 4(10):1283-1294

12. Tu Z: Eight novel families of miniature inverted repeat transposable elements in the African malaria mosquito, Anopheles gambiae. Proc Natl Acad Sci USA 2001, 98(4):1699-1704.

13. Jiang $N$, Feschotte $C$, Zhang $X$, Wessler SR: Using rice to understand the origin and amplification of miniature inverted repeat transposable elements (MITEs). Curr Opin Plant Biol 2004, 7(2):115-119.

14. Oki N, Yano K, Okumoto Y, Tsukiyama T, Teraishi M, Tanisaka T: A genomewide view of miniature inverted-repeat transposable elements (MITEs) in rice rice, Oryza sativa ssp japonica. Genes Genet Syst 2008, 83(4):321-329.

15. Benjak A, Boue S, Forneck A, Casacuberta JM: Recent amplification and impact of MITEs on the genome of grapevine (Vitis vinifera L.). Genome Biol Evol 2009, 1:75-84

16. Lu C, Chen J, Zhang Y, Hu Q, Su W, Kuang H: Miniature Inverted-Repeat Transposable Elements (MITEs) Have Been Accumulated through Amplification Bursts and Play Important Roles in Gene Expression and Species Diversity in Oryza sativa. Mol Biol Evol 2012, 29(3):1005-1017.

17. Mo YJ, Kim KY, Shin WC, Lee GM, Ko JC, Nam JK, Kim BK, Ko JK, Yu Y, Yang TJ: Characterization of Imcrop, a Mutator-like MITE family in the rice genome. Genes Genomics 2012, 34(2):189-198.

18. Yang G, Lee $Y H$, Jiang $Y$, Shi $X$, Kertbundit S, Hall TC: A two-edged role for the transposable element Kiddo in the rice ubiquitin2 promoter. Plant Cell 2005, 17(5):1559-1568.

19. Naito K, Zhang F, Tsukiyama T, Saito H, Hancock CN, Richardson AO, Okumoto Y, Tanisaka T, Wessler SR: Unexpected consequences of a sudden and massive transposon amplification on rice gene expression. Nature 2009, 461(7267):1130-1134.

20. Piriyapongsa J, Jordan IK: Dual coding of siRNAs and miRNAs by plant transposable elements. RNA 2008, 14(5):814-821.

21. Kuang H, Padmanabhan C, Li F, Kamei A, Bhaskar PB, Ouyang S, Jiang J, Buell CR, Baker B: Identification of miniature inverted-repeat transposable elements (MITEs) and biogenesis of their siRNAs in the Solanaceae: new functional implications for MITEs. Genome Res 2009, 19(1):42-56.

22. Lisch D, Bennetzen JL: Transposable element origins of epigenetic gene regulation. Curr Opin Plant Biol 2011, 14(2):156-161.

23. Casa AM, Brouwer C, Nagel A, Wang L, Zhang Q, Kresovich S, Wessler SR: The MITE family heartbreaker ( $\mathrm{Hbr})$ : molecular markers in maize. Proc Natl Acad Sci USA 2000, 97(18):10083-10089.

24. Lyons M, Cardle L, Rostoks N, Waugh R, Flavell AJ: Isolation, analysis and marker utility of novel miniature inverted repeat transposable elements from the barley genome. Molecular genetics genomics 2008, 280(4):275-285.

25. Wang $X$, Wang $H$, Wang J, Sun R, Wu J, Liu S, Bai Y, Mun JH, Bancroft I, Cheng F, et al: The genome of the mesopolyploid crop species Brassica rapa. Nat Genet 2011, 43(10):1035-1039.

26. Yang TJ, Kim JS, Kwon SJ, Lim KB, Choi BS, Kim JA, Jin M, Park JY, Lim MH, $\mathrm{Kim} \mathrm{HI}$, et al: Sequence-level analysis of the diploidization process in the triplicated FLOWERING LOCUS C region of Brassica rapa. Plant Cell 2006, 18(6):1339-1347.

27. Mun JH, Kwon SJ, Yang TJ, Seol YJ, Jin M, Kim JA, Lim MH, Kim JS, Baek S, Choi BS, et al: Genome-wide comparative analysis of the Brassica rapa gene space reveals genome shrinkage and differential loss of duplicated genes after whole genome triplication. Genome Biol 2009, 10(10):R111.

28. Zhang X, Wessler SR: Genome-wide comparative analysis of the transposable elements in the related species Arabidopsis thaliana and Brassica oleracea. Proc Natl Acad Sci USA 2004, 101(15):5589-5594.

29. Kapitonov W, Jurka J: Molecular paleontology of transposable elements from Arabidopsis thaliana. Genetica 1999, 107(1-3):27-37.

30. Johnston JS, Pepper AE, Hall AE, Chen ZJ, Hodnett G, Drabek J, Lopez R, Price HJ: Evolution of genome size in Brassicaceae. Ann Bot 2005, 95(1):229-235 
31. Feng C, Shengyi L, Jian W, Lu F, Silong S, Bo L, Pingxia L, Wei H, Xiaowu W: $B R A D$, the genetics and genomics database for Brassica plants. BMC Plant Biol 2011, 11(136):1-6.

32. Jiang N, Bao Z, Zhang X, Hirochika H, Eddy SR, McCouch SR, Wessler SR: An active DNA transposon family in rice. Nature 2003, 421(6919):163-167.

33. Yang G, Zhang F, Hancock CN, Wessler SR: Transposition of the rice miniature inverted repeat transposable element mPing in Arabidopsis thaliana. Proc Natl Acad Sci USA 2007, 104(26):10962-10967.

34. Casacuberta JM, Santiago N: Plant LTR-retrotransposons and MITEs: control of transposition and impact on the evolution of plant genes and genomes. Gene 2003, 311:1-11.

35. Yang G, Nagel DH, Feschotte C, Hancock CN, Wessler SR: Tuned for transposition: molecular determinants underlying the hyperactivity of a Stowaway MITE. Science 2009, 325(5946):1391-1394.

36. Rana D, Boogaart T, O'Neill CM, Hynes L, Bent E, Macpherson L, Park JY, Lim YP, Bancroft I: Conservation of the microstructure of genome segments in Brassica napus and its diploid relatives. Plant J 2004, 40(5):725-733.

37. Truco MJ, Hu J, Sadowski J, Quiros CF: Inter- and intra-genomic homology of the Brassica genomes: Implications for their origin and evolution. Theor Appl Genet 1996, 93(8):1225-1233.

38. Sarilar V, Marmagne A, Brabant P, Joets J, Alix K: BraSto, a Stowaway MITE from Brassica: recently active copies preferentially accumulate in the gene space. Plant Mol Biol 2011, 77(1-2):59-75.

39. Fontdevila A: Hybrid genome evolution by transposition. Cytogenet Genome Res 2005, 110(1-4):49-55.

40. Hu G, Hawkins JS, Grover CE, Wendel JF: The history and disposition of transposable elements in polyploid Gossypium. National Research Council Canada = Genome Conseil national de recherches Canada 2010, 53(8):599-607.

41. Santiago N, Herraiz C, Goni JR, Messeguer X, Casacuberta JM: Genome-wide analysis of the Emigrant family of MITEs of Arabidopsis thaliana. Mol Biol Evol 2002, 19(12):2285-2293.

42. Chen Y, Zhou F, Li G, XU Y: MUST: a system for identification of miniature inverted-repeat transposable elements and applications to Anabaena variabilis and Haloquadratum walsbyi. Gene 2009, 436(1-2):1-7.

43. Crooks GE, Hon G, Chandonia JM, Brenner SE: WebLogo: a sequence logo generator. Genome Res 2004, 14(6):1188-1190

44. Zuker M: Mfold web server for nucleic acid folding and hybridization prediction. Nucleic Acids Res 2003, 31(13):3406-3415.

45. Altschul SF, Gish W, Miller W, Myers EW, Lipman DJ: Basic local alignment search tool. J Mol Biol 1990, 215(3):403-410.

46. Ayele M, Haas BJ, Kumar N, Wu H, Xiao Y, Van Aken S, Utterback TR, Wortman JR, White OR, Town CD: Whole genome shotgun sequencing of Brassica oleracea and its application to gene discovery and annotation in Arabidopsis. Genome Res 2005, 15(4):487-495.

47. Tamura K, Peterson D, Peterson N, Stecher G, Nei M, Kumar S: MEGA5: Molecular Evolutionary Genetics Analysis using Maximum Likelihood, Evolutionary Distance, and Maximum Parsimony Methods. Mol Biol Evol 2011.

48. Lee SC, Lim MH, Kim JA, Lee SI, Kim JS, Jin M, Kwon SJ, Mun JH, Kim YK, $\mathrm{Kim} \mathrm{HU}$, et al: Transcriptome analysis in Brassica rapa under the abiotic stresses using Brassica $24 \mathrm{~K}$ oligo microarray. Mol Cells 2008, 26(6):595-605

49. Doyle J, Doyle J: A rapid DNA isolation procedure for small quantities of fresh leaf tissue. Phytochem Bull 1987, 19:11-15.

50. Rozen S, Skaletsky H: Primer3 on the WWW for general users and for biologist programmers. Methods Mol Biol 2000, 132:365-386.

51. Rohlf F: NTSYS-pc, version 2.10 z. Setauket, New York: Exeter Software; 2002.

doi:10.1186/1471-2229-13-56

Cite this article as: Sampath et al:: Characterization of a new high copy Stowaway family MITE, BRAMI-1 in Brassica genome. BMC Plant Biology 2013 13:56.

\section{Submit your next manuscript to BioMed Central and take full advantage of:}

- Convenient online submission

- Thorough peer review

- No space constraints or color figure charges

- Immediate publication on acceptance

- Inclusion in PubMed, CAS, Scopus and Google Scholar

- Research which is freely available for redistribution

Submit your manuscript at www.biomedcentral.com/submit
Biomed Central 